\title{
Analysis of Inelasticity Effect Due to Damage on Stress Distributions in Composite Laminates
}

\author{
C. L. CHOw* \\ Department of Mechanical Engineering \\ University of Michigan-Dearborn \\ Dearborn, Michigan 48128-1491 \\ F. YANG** AND A. ASUNDI \\ Department of Mechanical Engineering \\ University of Hong Kong \\ Hong Kong
}

\begin{abstract}
A damage mechanics model characterizing damage behavior of composite materials proposed earlier by the authors is employed to analyze the damage effects on stress field near the free edge in symmetrically laminated graphite/epoxy composites of finite dimensions under uniaxial tension. A quasi-three-dimensional finite element analysis is developed for the present investigation. The results from the damaged and undamaged stress distributions of $\left[0 / 90^{\circ}\right]_{s},\left[90 / 0^{\circ}\right]_{s}$, and $\left[ \pm 45^{\circ}\right]_{s}$ laminates are compared and examined. The processes of initiation and development of damage zone in these composite laminates are also discussed.
\end{abstract}

\section{INTRODUCTION}

$\mathbf{E}$ XTENSIVE ACCEPTANCE AND utilization of fiber reinforced composite laminates in aerospace and aircraft structures necessitates a proper and rational analysis of their mechanical properties, especially their failure characteristics. The ability to predict accurately the strength of a particular composite as well as its load carrying capacity is important for safe design. Researchers in the past have revealed that in realistic engineering situations, various material failure mechanisms, which are usually categorized into four distinct types: matrix cracking, fiber-matrix interface debonding, interlaminar delamination and fiber fracture, have been observed individually or simultaneously in fiber reinforced composite laminates during varying operation processes. These micro-failure events, commonly considered as damage, cause degradation in several global material

\footnotetext{
*Author to whom correspondence should be addressed.

**Currently on leave from Huazhong University of Science \& Technology, Wuhan, China.
} 
properties, such as reducing the strength and stiffness of the material, as well as detriment to the life of the structure.

Since the damage in composite laminates is complex, various, and intricately related to a variety of modes under different circumstances (as discussed in Reference [1]), besides the inherent anisotropy and heterogeneity of composites, it is a formidable task to study microscopically the fine details of the failure behaviors of the materials. Fortunately, as the internal damage in laminates is noncatastrophic in many cases, it is rational to use a systematic theory with homogenization techniques to investigate the locally averaged effect of the damage on macrophysical response. This phenomenological approach is usually referred to as damage mechanics, where the influence of micro-cracks represented by a number of internal state variables (called damage parameters) is reflected in constitutive relations of the material, rather than boundary conditions, which is different from the fracture mechanics concepts. For example, under certain operating conditions, a composite material may exhibit nonlinear properties before its final failure, instead of the simple elasticity commonly assumed based on the classical laminated plate theory (CLPT). This inelasticity, from the point of view of damage mechanics, is considered in general to be related to the irreversible thermodynamics processes involving energy dissipation and stiffness variation primarily caused by the presence of micro-structure changes of the material (see References [2-5]). Taking this nonlinear behavior of composite laminates into account, an improved design analysis capable of characterizing accurate response of composite structures can be realized.

It has long been recognized that at free edges in a finite-width composite laminate, the interlaminar stresses arise due to mismatch in material properties between plies. According to past investigators, significant interlaminar shear and normal stresses are found to exist at the vicinity of the free edge when the laminate is subjected to in-plane loads. These high interlaminar stresses can lead to delamination, a critical failure mechanism for laminated composite materials, causing failure in the laminate at in-plane loads much lower than the loads at which the laminate would fail if only in-plane fracture were the failure mechanism. Thus, in this region near the laminate free edge, there exists a boundary layer where the state of stress is three-dimensional. The CLPT, although adequate for interior areas removed from laminate geometric discontinuities, such as free edges, is no longer valid, as it permits one to define effective elastic properties of the laminate as a whole, and to compute integrated values of the in-plane stress components across the laminate thickness based on the assumption of planar state of stress within each lamina of a multidirectional thin laminate.

It is well known that it is not possible to gain an understanding and general characterization of variously insidious damage mechanisms in composite laminates in the absence of an accurate means of calculating those interlaminar stress components with high gradients in the neighborhood of the free edge. Numerous investigations have thus been performed to obtain three-dimensional stress distributions using different methods, including the finite difference scheme [6], perturbation theory [7,8], complex stress potentials and eigen-function series [9], 
Reissner's variational principle [10], the global-local model [11], the asymptotic technique [12], the finite element method [13-15] and the hybrid finite element method [16,17]. Although most of these investigations are limited to materials with linear elastic properties, the inelastic characteristics of composite materials require further study due to the complexity of the problem.

In order to have reliable predictions of displacement and stress fields, it is necessary that the free edge stress calculation be able to approximate the real situation as closely as possible. Moreover, the computational technique should be simple so as to be easily mastered. After examining available computational approaches, the displacement-based finite element method is considered to be a feasible and practical alternative because of its versatility in handling nonhomogeneous material properties, irregular specimen shapes, complex boundary conditions and general loading. An important advantage relevant to the analysis is that the inelastic characteristics of the composite material can be easily implemented into its solution procedures. It is reasonable to expect that if the nonlinearity due to damage described in References [2-5] is included in the evaluation of stresses in the free edge region, the singular behaviors of interlaminar stresses postulated under the assumption of linear elasticity would be altered.

The purpose of this study is to examine the damage effects on the interlaminar stress distribution near the free edge when the composite laminate is subjected to a uniformly loaded uniaxial tension. The model proposed in References [4] and [5], which describes the damage behavior of brittle composite materials, is extended and applied to the study. A quasi-three-dimensional finite element program is developed to aid the present analysis. A comparison of the results for the graphite/epoxy $\left[0 / 90^{\circ}\right]_{s},\left[90 / 0^{\circ}\right]_{s}$, and $\left[ \pm 45^{\circ}\right]_{s}$ laminates with and without damage are included. The initiation and growth of the damage zone, a specific form of the failure mode of these laminates, are also investigated.

\section{INELASTIC MODEL FOR COMPOSITE LAMINA WITH DAMAGE}

There are various methods of analysis currently available in assessing damage properties of composite materials (see References [18-21]). The anisotropic model of damage mechanics by Chow and Yang $[4,5]$ is chosen for this investigation.

As elucidated in Reference [5], at a certain damage state $D$, the stress at a point in the material is $\sigma_{i}$, with its corresponding reversible strain $\epsilon_{i}(i=1,2, \ldots, 6$, as the contracted notation and summation convention are adopted). Because of the characteristic unloading behavior of brittle composite material with damage, the stress and strain may be related using generalized Hooke's law as

$$
\epsilon_{i}=C_{i j} \sigma_{\jmath} \quad(D \text { fixed })
$$

where $C_{t j}=C_{j i}$ represent the elastic compliance components at current state. These material coefficients reflecting progressive damage will change during the loading process. 
Generally, the total reversible strain increment in damaging process is

$$
d \epsilon_{i}=C_{i j} d \sigma_{j}+d C_{i j} \sigma_{j}
$$

which can be divided into two parts. The component of damaged strain increment, the second term on the right-hand side of the equation (which should be absent during elastic loading or unloading), is referred to as

$$
d \epsilon_{i}^{d}=d C_{i j} \sigma_{j}
$$

Similarly, the quantity

$$
d \epsilon_{\imath}^{e}=C_{i j} d \sigma_{j}
$$

can be considered as the elastic strain increment.

Equations (1) and (2) give the description of the elastic and damaged responses of the composite materials. However, before these equations can be employed, we need to establish a damage criterion in distinguishing between the elastic and damage behaviors. To this end, the damage surface is defined as

$$
F=F\left(\sigma_{i}, D\right)=0
$$

which encloses a region in stress space, and contains all possible loading processes such that the material behaviors are linearly elastic without further change in the material properties. As the damage develops, this area expands to encompass those additional state points that can be reached by elastic reloading with the new material coefficients because of its earlier damaging process. The physical significance of this function is analogous to that of the yielding surface in the theory of plasticity.

Moreover, a damage potential function $G$ in stress space is taken to have the following form:

$$
G=G\left(\sigma_{i}, D\right)=0
$$

Then, the damaged strain increment can also be obtained by

$$
d \epsilon_{i}^{d}=d \lambda \frac{\partial G}{\partial \sigma_{i}}
$$

$d \lambda$ in Equation (7) is a scalar of proportionality. If the energy per unit volume dissipated during the damaging process is selected as damage variable $D$, its increment may be expressed as

$$
d D=\frac{1}{2} \sigma_{i} d \epsilon_{i}^{d}
$$


On the bases of the hypothesis that the material compliance $C_{i j}$ only depends on the damage variable $D$ (not directly relevant to individual stress components), and the condition that any stress increment coincident within the damage surface will not lead to the changes of $\epsilon_{i}^{d}$ as well as $C_{i j}[2,3]$, the expression that relates the variation of $C_{i j}$ to the damage surface $F$ and the damage potential $G$ during damaging process, by using Equations (3) and (5)-(8), can be formulated as $[4,5]$

$$
\begin{aligned}
C_{i j}^{\prime} & =\frac{2}{T}\left\{\frac{\partial^{2} G}{\partial \sigma_{i} \partial \sigma_{j}}-\frac{1}{T} \frac{\partial G}{\partial \sigma_{\imath}} \frac{\partial G}{\partial \sigma_{j}}\right. \\
& -\frac{1}{2 T}\left(\frac{\partial G}{\partial \sigma_{i}} \sigma_{u} \frac{\partial^{2} G}{\partial \sigma_{u} \partial \sigma_{j}}+\frac{\partial G}{\partial \sigma_{j}} \sigma_{u} \frac{\partial^{2} G}{\partial \sigma_{u} \partial \sigma_{i}}\right) \\
& -\frac{1}{2 P}\left[\frac{\partial F}{\partial \sigma_{j}}\left(\frac{\partial^{2} G}{\partial \sigma_{i} \partial \sigma_{m}} \sigma_{m}-2 \frac{\partial G}{\partial \sigma_{i}}-\frac{1}{T} \frac{\partial G}{\partial \sigma_{i}} \frac{\partial^{2} G}{\partial \sigma_{r} \partial \sigma_{p}} \sigma_{r} \sigma_{p}\right)\right. \\
& \left.\left.+\frac{\partial F}{\partial \sigma_{i}}\left(\frac{\partial^{2} G}{\partial \sigma_{j} \partial \sigma_{m}} \sigma_{m}-2 \frac{\partial G}{\partial \sigma_{j}}-\frac{1}{T} \frac{\partial G}{\partial \sigma_{j}} \frac{\partial^{2} G}{\partial \sigma_{r} \partial \sigma_{p}} \sigma_{r} \sigma_{p}\right)\right]\right\}
\end{aligned}
$$

in which

$$
C_{i j}^{\prime}=\frac{d C_{i j}}{d D}, \quad P=\frac{\partial F}{\partial \sigma_{u}} \sigma_{u}, \quad T=\frac{\partial G}{\partial \sigma_{u}} \sigma_{u}
$$

$C_{i j}^{\prime}$ can be determined as long as the expressions of functions $F$ and $G$ are known. As a specific case, let $G=F$ and consider the damage surface

$$
F\left(\sigma_{i}, D\right)=\left(R_{i j} \sigma_{i} \sigma_{j}\right)^{1 / 2}-K(D)=0
$$

where $K(D)$ is a state function and changes with the development of damage $D$, and the components in $R_{i j}$ (without loss generality, it may be assumed that $R_{i j}=R_{j i}$ ) can be evaluated from experiments. $F$ is defined in such a way that the hydrostatic stress will contribute to damage process.

Furthermore, the equivalent stress $\sigma_{o}$ and the equivalent damaged strain increment $d \epsilon_{o}^{d}$ are defined as

$$
\begin{gathered}
\sigma_{o}^{2}=R_{i j} \sigma_{i} \sigma_{j} \\
d D=\frac{1}{2} \sigma_{i} d \epsilon_{i}^{d}=\frac{1}{2} \sigma_{o} d \epsilon_{o}^{d}
\end{gathered}
$$

and $\sigma_{o}-\epsilon_{o}^{d}$ relation established from experiment is

$$
\epsilon_{o}^{d}=a \sigma_{o}^{b}-a \sigma_{s}^{b}
$$


where $a$ and $b$ are material constants, and $\sigma_{s}$ is the equivalent stress at the threshold of damage. As $C_{t}(0)$ denotes the compliance of the material element without damage, the equation for the change of $C_{i j}$ results in [5]

$$
C_{i j}(D)=C_{i j}(0)+\frac{a b}{(b-1)}\left(\sigma_{o}^{b-1}-\sigma_{s}^{b-1}\right) R_{i j}
$$

and

$$
D=\frac{a b}{2(b+1)}\left(\sigma_{o}^{b+1}-\sigma_{s}^{b+1}\right) \quad\left(\text { for } d \sigma_{o} \geq 0\right)
$$

Equation (15) relates the rate of damage progression with stress, and Equation (14) can be employed to obtain instantaneous $C_{i j}(D)$ during a damage-induced loading process. It is evident that the coefficients $R_{i j}$ actually reflect the anisotropic behavior caused by material damage. In a certain unloading-reloading cycle, $C_{i j}(D)$ are constants, as $D$ equals the value determined from $\sigma_{o}$ at the beginning of unloading. Upon reloading, $D$ remains invariant and so does the $C_{i j}(D)$, unless $\sigma_{o}$ exceeds its preceding maximum value.

The establishment of the elastic-damaged tangent stiffness matrix provides a rational basis for the incremental finite element approach applicable to the investigation of composite laminates with damage. The procedure for solving the corresponding finite element equations is similar to the conventional nonlinear analysis. The main difference lies in the stress-strain relations, which currently include a damage variable. Therefore, the modification of the stiffness matrix during calculation is necessary.

From Equations (1)-(4), one obtains

$$
d \sigma_{i}=S_{i j}\left(d \epsilon_{j}-d \epsilon_{j}^{d}\right)
$$

Equation (5) and its associated consistency condition give

$$
d F=\frac{\partial F}{\partial \sigma_{i}} d \sigma_{i}+\frac{\partial F}{\partial D} d D=0
$$

Considering Equations (16) and (8), Equation (17) becomes

$$
\frac{\partial F}{\partial \sigma_{i}} S_{i j}\left(d \epsilon_{j}-d \epsilon_{j}^{d}\right)+\frac{1}{2} \frac{\partial F}{\partial D} \sigma_{i} d \epsilon_{i}^{d}=0
$$

With Equation (7), the scalar of proportionality $d \lambda$ can be determined as

$$
d \lambda=\frac{\frac{\partial F}{\partial \sigma_{k}} S_{k l} d \epsilon_{l}}{\frac{\partial F}{\partial \sigma_{m}} S_{m n} \frac{\partial G}{\partial \sigma_{n}}-\frac{1}{2} \frac{\partial F}{\partial D} \sigma_{m} \frac{\partial G}{\partial \sigma_{m}}}
$$


Hence,

$$
d \epsilon_{\imath}^{d}=\frac{\frac{\partial G}{\partial \sigma_{\imath}} \frac{\partial F}{\partial \sigma_{k}} S_{k l} d \epsilon_{l}}{\frac{\partial F}{\partial \sigma_{m}} S_{m n} \frac{\partial G}{\partial \sigma_{n}}-\frac{1}{2} \frac{\partial F}{\partial D} \sigma_{m} \frac{\partial G}{\partial \sigma_{m}}}
$$

By substituting Equation (20) into Equation (16), the relationship between the elastic-damaged stress increments $d \sigma_{i}$ and the total reversible strain increments $d \epsilon_{i}$ can be finalized as

$$
d \sigma_{i}=S_{i j}^{e d} d \epsilon_{j}
$$

where $S_{i j}^{e d}$ is the elastic-damaged instantaneous tangent modulus matrix; it assumes the following form

$$
S_{i j}^{e d}=S_{i j}-\frac{S_{i k} \frac{\partial G}{\partial \sigma_{k}} \frac{\partial F}{\partial \sigma_{l}} S_{l j}}{\frac{\partial F}{\partial \sigma_{m}} S_{m n} \frac{\partial G}{\partial \sigma_{n}}-\frac{1}{2} \frac{\partial F}{\partial D} \sigma_{m} \frac{\partial G}{\partial \sigma_{m}}}
$$

It should be noted that the $S_{i j}^{e d}$ in this equation are not constants like those in plasticity theory. They are dependent upon the previous loading histories that involve damage progression. Equation (22) is obviously important in numerical analysis of damage response of the structure of composite material, especially when employing the finite element method.

When $G=F$ and $F$ takes the form of Equation (10), after some manipulation [using Equations (10) and (13)], Equation (22) is finally expressed as

$$
S_{i j}^{e d}=S_{i j}(D)-\frac{a b \sigma_{o}^{b-3} S_{i p}(D) R_{p q} \sigma_{q} R_{m n} \sigma_{n} S_{m j}(D)}{1+a b \sigma_{o}^{b-3} R_{s t} \sigma_{s} S_{t l}(D) R_{l k} \sigma_{k}}
$$

and $\left[S_{i j}(D)\right]=\left[C_{i j}(D)\right]^{-1}$, if the inverse exists.

From the previous discussions, it may be concluded that Equations (1), (2) and (21) are sufficient to describe the damage behavior of brittle composite materials, as long as the material coefficients in Equations (11) and (13) are determined from experimental results.

\section{ASSUMPTIONS AND FINITE ELEMENT FORMULATION}

To show the influence of damage on the response of composite structures, consider a rectangular composite laminate containing four unidirectional fiber reinforced layers of uniform thicknesses which are symmetrically stacked about the midplane. The composite laminate of width $2 b$ and total thickness of $4 h$ is subjected to a uniform axial extension strain $\epsilon_{o}$ in the $x$-direction, as shown in Figure 


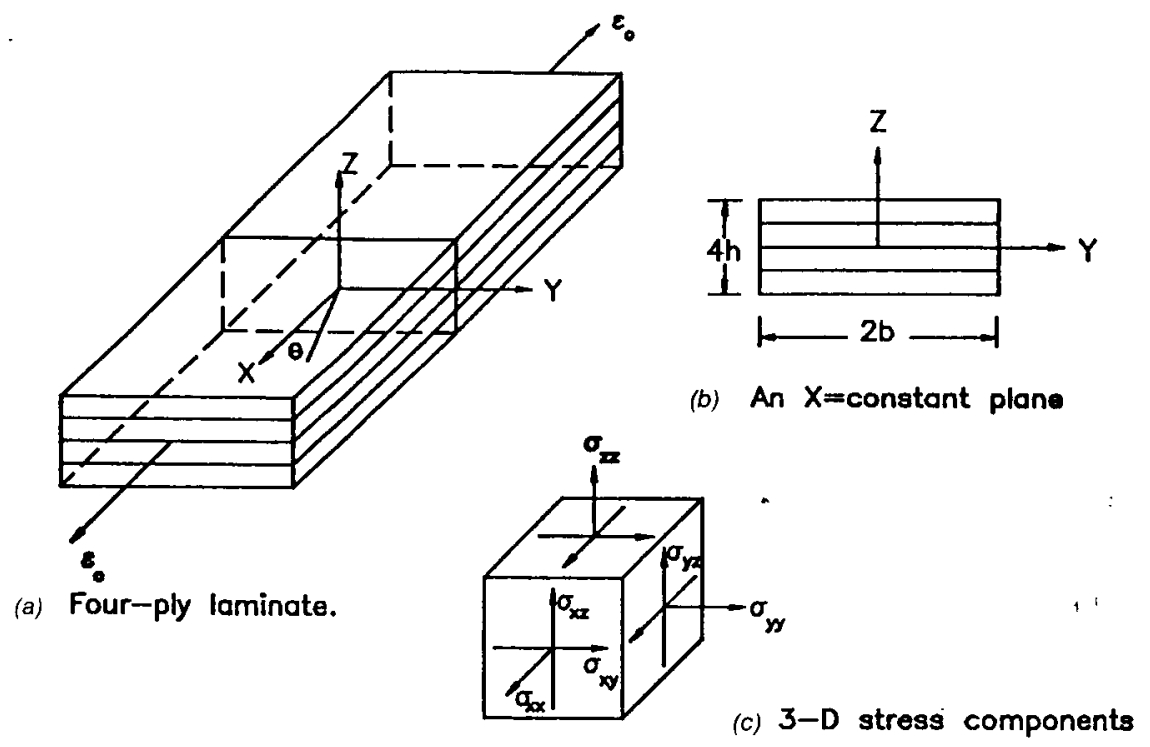

Figure 1. Laminate configuration.

1. Away from the loading ends, if the laminate is sufficiently long, the displacements $u, v, w$ in the $x, y, z$ directions in any $x=$ constant plane can be assumed to be independent of $x$. This implies that the three components of displacement have the form as follows:

$$
\begin{aligned}
& u(x, y, z)=\epsilon_{o} x+U(y, z) \\
& v(x, y, z)=V(y, z) \\
& w(x, y, z)=W(y, z)
\end{aligned}
$$

where $\epsilon_{o}$ is the uniform axial strain, and $U, V$, and $W$ are functions of the coordinates $y$ and $z$ alone. The problem is quasi-three-dimensional in that all six components of stress and strain may be non-zero, but the analysis can be restricted to a generic two-dimensional cross section. Because of symmetry with respect to the midplane, only half of the laminate needs to be considered. However, for the purpose of computation, further simplification to one-quadrant laminate is possible by imposing appropriate symmetry conditions along the longitudinal midplane. The boundary conditions associated with the quarter section of the laminate $(0 \leq y \leq b ; 0 \leq z \leq 2 h)$ are $U(0, z)=V(0, z)=W(y, 0)=0$, $\sigma_{y y}(b, z)=\sigma_{x y}(b, z)=\sigma_{y z}(b, z)=0$ along the free edge, and $\sigma_{z x}(y, 2 h)=$ $\sigma_{y z}(y, 2 h)=\sigma_{x z}(y, 2 h)=0$ on the top surface. 
In the analyses, the following assumptions are employed:

1. Each layer is modeled as a macroscopically homogeneous but orthotropic material. Individual fibers and matrix are not considered.

2. The laminae are of equal thickness and perfectly bonded together. No adhesive layer is included.

3. Time and rate effects are neglected and environmental effects such as temperature and moisture are excluded.

4. The laminate is in an initially free stress state and body force is ignored.

5. Deformation is small, the material without damage is linearly elastic, and its response can be described by generalized Hooke's law.

With the above assumptions, the constitutive model developed to describe the nonlinearity induced by material damage can be implemented into a finite element analysis to study the stress distribution of composite laminates with straight free edge along the $x$-direction at $y=b$ (see Figure 1).

In the finite element analysis, the laminate is modeled in the $y-z$ plane based on the condition specified in Equation (24) for which the quadrilateral isoparametric finite element is chosen. Figure 2(a) depicts a typical quadrilateral isoparametric element with nodes 1-8. This element is presented in a global coordinate system. Another coordinate system, called the natural coordinate system or local coordinate system, which will allow a simple square element to be mapped into the $y-z$ system and still satisfy the continuity requirements, is also introduced. The simple square element and the local coordinate system are shown in Figure 2(b).

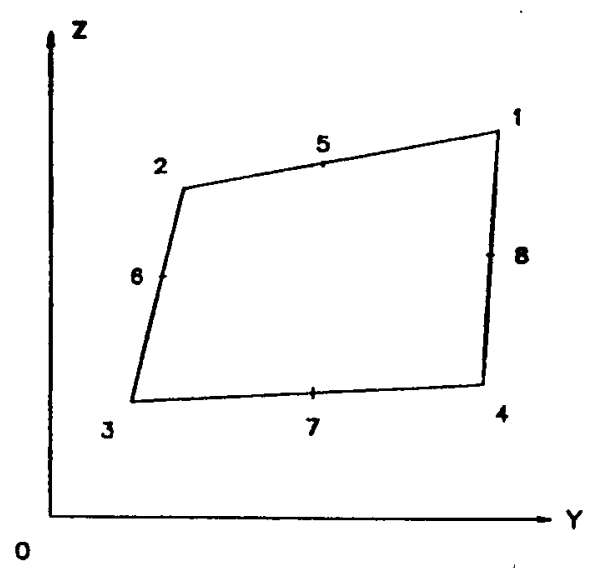

(a) Global coordinate system

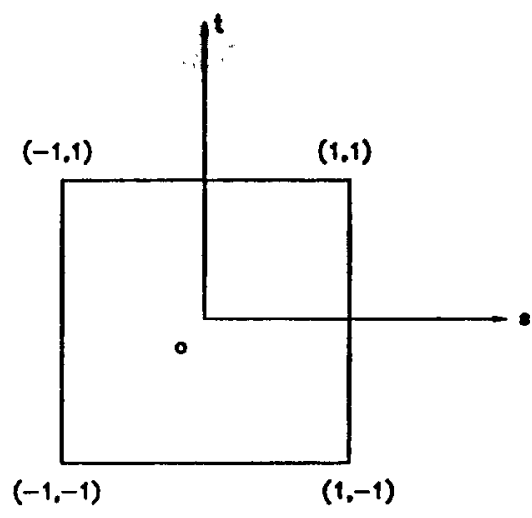

(b) Local coordinate syatem

Figure 2. A typical isoparametric finite element. 
From the displacement conditions specified in Equation (24), the strain components are defined as

$$
\begin{aligned}
& \epsilon_{x x}=\frac{\partial u}{\partial x}=\epsilon_{o}, \quad \epsilon_{y y}=\frac{\partial v}{\partial y}, \quad \epsilon_{z z}=\frac{\partial w}{\partial z}, \quad \gamma_{y z}=\frac{\partial v}{\partial z}+\frac{\partial w}{\partial y}, \\
& \gamma_{x z}=\frac{\partial u}{\partial z}+\frac{\partial w}{\partial x}=\frac{\partial u}{\partial z}, \quad \gamma_{x y}=\frac{\partial u}{\partial y}+\frac{\partial v}{\partial x}=\frac{\partial u}{\partial y}
\end{aligned}
$$

The strain-displacement relationships for the quasi-three-dimensional analysis can therefore be written as

$$
\{\epsilon\}=\{\epsilon\}_{0}+[B]\{U\}
$$

where

$$
\{\epsilon\}_{o}=\left\{\epsilon_{o} 00000\right\}^{r}
$$

From the formulation of the eight-node isoparametric element described in Reference [22], the $[B]$ matrix shown in Equation (26) can be readily established as

$$
[B]=\left[\begin{array}{ccccccccc}
00 & 000 & 000 & 000 & 000 & 000 & 000 & 000 & 0 \\
0 b_{1} & 00 b_{2} & 00 b_{3} & 00 b_{4} & 00 b_{5} & 00 b_{6} & 00 b_{7} & 00 b_{8} & 0 \\
00 & c_{1} 00 & c_{2} 00 & c_{3} 00 & c_{4} 00 & c_{5} 00 & c_{6} 00 & c_{7} 00 & c_{8} \\
0 c_{1} & b_{1} 0 c_{2} & b_{2} 0 c_{3} & b_{3} 0 c_{4} & b_{4} 0 c_{5} & b_{5} 0 c_{6} & b_{6} 0 c_{7} & b_{7} 0 c_{8} & b_{8} \\
c_{1} 0 & 0 c_{2} 0 & 0 c_{3} 0 & 0 c_{4} 0 & 0 c_{5} 0 & 0 c_{6} 0 & 0 c_{7} 0 & 0 c_{8} 0 & 0 \\
b_{1} 0 & 0 b_{2} 0 & 0 b_{3} 0 & 0 b_{4} 0 & 0 b_{5} 0 & 0 b_{6} 0 & 0 b_{7} 0 & 0 b_{8} 0 & 0
\end{array}\right]
$$

in which

$b_{i}=P_{1 i} \cdot \overline{J_{1}}+P_{2 i} \cdot \overline{J_{2}}, \quad C_{i}=P_{1 i} \cdot \overline{J_{3}}+P_{2 i} \cdot \overline{J_{4}}$

where $\bar{J}_{i}$ is the inversed Jacobian matrix component and $P_{i j}$ are the functions related to the local displacement derivatives to the nodal displacements. It is clear that $[B]$ is a matrix depending only upon the geometry of the element and the point $(s, t)$ within the element where the strain is to be evaluated.

In an elastic-damaged analysis, because of the nonlinear relationship between the stress $\{\sigma\}$ and the strain $\{\epsilon\}$ as shown in Section 2, the stress in an element is a nonlinear function of strains, and therefore, is a nonlinear function of the nodal displacement $\{U\}$. To acquire the solution for $\{U\}$ corresponding to a given set of external conditions, the iterative processes usually have to be employed. Moreover, an incremental analysis is necessary in order to trace the change of displacement, strain and stress components, and the actual variation of the applied conditions. For an elastic-damaged process, if the displacement $\{U\}_{n}$, stress $\{\sigma\}_{n}$ and $\{\epsilon\}_{n}$ at the $n$th step are known, the displacement increment $\{\Delta U\}$ can be deter- 
mined from the following equations by using the virtual work principle, as long as the $\epsilon_{o}$ and $\Delta \epsilon_{o}$ are prescribed

$$
\begin{gathered}
{[K]_{n} \cdot\{\Delta U\}=-\Delta \epsilon_{o} \cdot[K x]_{n}^{T}-\{F\}_{n}} \\
\{F x\}_{n+1}=[K x]_{n} \cdot\{\Delta U\}+\{F x\}_{n}+\Delta \epsilon_{o} \cdot[K o x]_{n}
\end{gathered}
$$

where

$$
\begin{aligned}
{[K]_{n} } & =\int_{\substack{\text { area } \\
y-=}}[B]^{T} \cdot\left[S^{e d}\right] \cdot[B] d A \\
{[K X]_{n} } & =\int_{\substack{\text { arca } \\
y-=}}[B]^{T} \cdot\left[S^{e d}\right] \cdot\{Q\} d A \\
\{F\}_{n} & =\int_{\substack{\text { area } \\
y-z}}[B]^{T} \cdot\{\sigma\}_{n} d A \\
\{F x\}_{n} & =\int_{\substack{\text { area } \\
y-z}}\{Q\}^{T} \cdot\{\sigma\}_{n} d A \\
{[K o x]_{n} } & =\int_{\substack{\text { area } \\
v-z}}\{Q\}^{T} \cdot\left[S^{e d}\right] \cdot\{Q\} d A
\end{aligned}
$$

and $\{Q\}^{T}=\{100000\},\left[S^{e d}\right]$ takes the form of Equation (23). Actually, $\epsilon_{o}$ and $\Delta \epsilon_{\mathrm{o}}$ play a role of external applied force, and the corresponding equivalent external nodal force in the $x$-direction $\{F x\}$ can be calculated from Equation (28). The strain and stress increments of the element can be easily derived from Equation (26) and (21),

$$
\begin{gathered}
\{\Delta \epsilon\}=\Delta \epsilon_{0}\{Q\}+[B]\{\Delta U\} \\
\{\Delta \sigma\}=\left[S^{e d}\right] \cdot\{\Delta \epsilon\}
\end{gathered}
$$

Therefore, the displacement $\{U\}_{n+1}$, stress $\{\sigma\}_{n+1}$ and $\{\epsilon\}_{n+1}$ at the $(n+1)$ th step are

$$
\{U\}_{n+1}=\{U\}_{n}+\{\Delta U\}
$$




$$
\begin{aligned}
& \{\epsilon\}_{n+1}=\{\epsilon\}_{n}+\{\Delta \epsilon\} \\
& \{\sigma\}_{n+1}=\{\sigma\}_{n}+\{\Delta \sigma\}
\end{aligned}
$$

Having determined the stiffness relation for each element, [Equation (27)], the overall structural stiffness for the composite laminate to be analyzed is formed in the usual way using the direct stiffness method.

The equations of finite element discretization enable the necessary modifications to be made on a three-dimensional finite element code to solve the stress distribution problem of composite laminates with straight free edge based on the proposed quasi-three-dimensional analysis. The nonlinearities caused by the elastic-damaged behavior of the composite laminate are handled by the modified Newton-Raphson method (MNRM) that has been widely used in the finite element iteration procedure. With the calculated $(i-1)$ th approximation, $\{U\}_{n}^{(i-1)}$, to the $\{U\}_{n}$, the corresponding iteration scheme for the MNRM is written as, for $i=1,2,3, \ldots$,

$$
\begin{gathered}
{[K]_{n}\{\Delta U\}^{(i)}=-\Delta \epsilon_{o} \cdot[K x]_{n+1}^{T}-\{F\}_{n+1}^{(i-1)}} \\
\{U\}_{n+1}^{(i)}=\{U\}_{n+1}^{(i-1)}+\{\Delta U\}^{(i)}
\end{gathered}
$$

with the initial conditions

$$
\{U\}_{n+1}^{(0)}=\{U\}_{n}, \quad\{U\}_{n+1}^{(0)}=\{U\}_{n}
$$

This iteration procedure continues until a proper convergence criterion is satisfied.

In the investigation, one quadrant of the cross section of the laminate is modeled by 160 conventional two-dimensional eight-node isoparametric elements. The mesh used is depicted in Figure 3 and is refined near the boundary of the laminate. In order to verify the developed computer program, it is executed first to calculate the linear-elastic interlaminar stresses. The results computed with the program are compared with those described in References [8] and [14], and excellent agreement has been achieved.

\section{RESULTS AND DISCUSSIONS}

For the present investigation, only two types of graphite/epoxy composite laminates, cross-ply and angle-ply, are studied. The reason for selecting these laminates is that the cross-ply laminate subjected to unixial strain shows mismatch in elastic deformation along the loading direction, while the angle-ply laminate displays mismatch in elastic shear properties.

Prior to using the finite element analysis to compute the nonlinear damage response of each laminate subjected to uniformly applied uniaxial loading, the basic mechanical properties of each lamina must first be determined. The experimental method developed for the determination of the virgin and damaged 


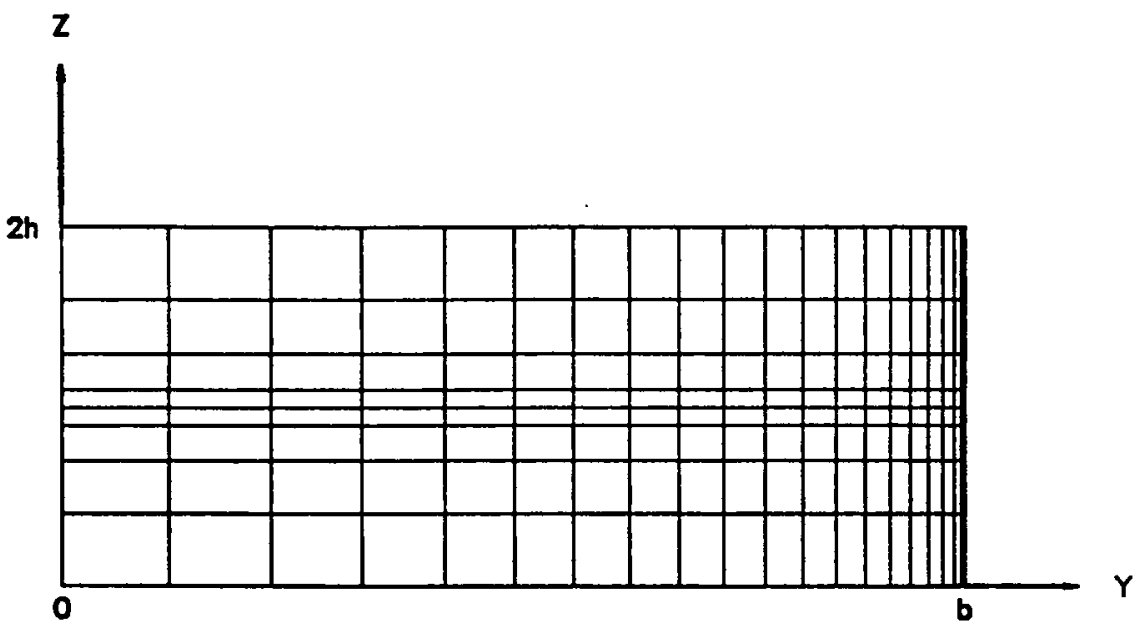

Figure 3. Finite element mesh for finite width laminate.

properties has been described in References [4] and [5], to which interested readers should refer for details. In essence, a series of unidirectional laminates of several orientations made of T300 graphite fiber in 648 epoxy matrix was loaded under uniaxial tension. The fiber volume fraction of the laminae chosen is about 65 percent, and the basic mechanical properties of virgin lamina are

$$
\begin{gathered}
E_{11}^{\circ}=1.25 \times 10^{5} \mathrm{MPa}, E_{22}^{\circ}=E_{33}^{\circ}=1.11 \times 10^{4} \mathrm{MPa} \\
\nu_{21}^{\circ}=\nu_{31}^{\circ}=0.03, \nu_{23}^{\circ}=0.338, G_{12}^{\circ}=G_{13}^{\circ}=G_{23}^{\circ}=3.3 \times 10^{3} \mathrm{MPa}
\end{gathered}
$$

where the subscripts 1,2 , and 3 correspond to the longitudinal, transverse and thickness directions, respectively, of a zero-degree composite lamina.

A second series of tests was performed for unidirectional laminates subjected to repeated loading and unloading conditions. This was done to establish the relationship between $\sigma_{o}$ and $\epsilon_{d}$ in Equation (13) from which the material constants of damage in the equation were evaluated as

$$
a=0.114 \times 10^{-4}, b=1.24, \sigma_{s}=9 \mathrm{MPa}
$$

In the determination of the non-vanishing constants of $R_{i j}$ in Equation (11), another set of unidirectional angled laminates of $22.5,45$, and 67.5 degrees was loaded under uniaxial tension. The test results established the relationship between the ply-angles and the $R_{i j}$, based on which the non-vanishing $R_{i j}$ values determined are

$$
R_{22}=R_{33}=1, R_{44}=R_{55}=R_{66}=1.9, R_{23}=0.05
$$


For the analysis of cross-ply laminates, $\left[0 / 90^{\circ}\right]_{s}$ and $\left[90 / 0^{\circ}\right]_{s}$ laminates are first considered, as they are simpler cross-ply laminates to be analyzed. The layers in these laminates are oriented at either 0 or 90 degrees and there are no off-axis plies. As these laminates subjected to uniaxial strain exhibit an elongation mismatch between plies along the loading direction, there are no in-plane and interlaminar shear stress components, $\sigma_{x y}$ and $\sigma_{x z}$, for both damaged and undamaged cases because of zero mismatch in the coefficient of mutual influence representing a coupling between axial and shear strain. The mismatch of Poisson's ratio between the adjacent dissimilar plies leads to different lateral strains, $\epsilon_{y y}$, in unbonded layers, but results in identical strains at the layer interfaces in perfectly bonded laminates with accompanying interlaminar stresses, $\sigma_{y y}$ and $\sigma_{y z}$.

For the case of the $\left[0 / 90^{\circ}\right]_{s}$ laminate, first damage is observed to occur relatively early in the loading process $\left(\epsilon_{0}=0.0006977\right)$, which takes place in an edge element of the $90^{\circ}$ layer near the interface. The damage zone spreads very rapidly in the $90^{\circ}$ lamina as the applied strain increases. Figure 4 displays several contours of damage zones in the laminate. Since the fibers are much stronger than the matrix, the damage zone is confined by the $0^{\circ}$ ply and will not develop inside it. However, the stresses in the $0^{\circ}$ layer will interact with the $90^{\circ}$ ply and redistribute because of the damage progress in $90^{\circ}$ ply. Figures 5-7 present the distribution of normal stresses $\sigma_{x x}, \sigma_{y y}$, and $\sigma_{z z}$, both inelastic and pseudo elastic, along the midplane of the laminate at $\epsilon_{0}=0.001$. Here, the pseudo elastic stresses are calculated from linear elastic theory with the original material stiffness without degradation. It can be shown from the figures that a substantial reduction of $\sigma_{x x}$ value results from the material stiffness degradation in the $x$-direction due to the damage process in the $90^{\circ}$ layer. However, there is no damage influence on the stress component $\sigma_{y y}$ as it is along the fiber direction of the $90^{\circ}$ layer. In Figure 7, although the distributions of the pseudo elastic and inelastic $\sigma_{z z}$ components are in similar shape, the maximum value of the damaged stress is reduced. The distributions of stress components $\sigma_{z z}$ and $\sigma_{y z}$ along the interface of the laminate for $\epsilon_{o}=0.001$ are shown in Figure 8 and Figure 9, respectively. For the given tension strain, very little difference may be found between the damaged and pseudo elastic stresses, as the influence of damage on the $0^{\circ}$ layer is not evident. However, the peak values of these stress components near the free edge due to damage are smaller than those pseudo elastic stresses. The comparisons between the damaged and pseudo elastic free edge stress components $\sigma_{z z}$ and $\sigma_{y z}$ for $\epsilon_{o}=0.001$ are depicted in Figure 10 and Figure 11, respectively. The influence of damage on these stress components is concentrated mainly inside the $90^{\circ} \mathrm{ply}$ and at the interface. The changes of these stress com ponents in the $0^{\circ}$ layer can be neglected, especially on the upper side of this ply.

For the $\left[90 / 0^{\circ}\right]_{s}$ laminate, damaging process is found to initiate in an edge element of the $90^{\circ}$ layer near the $90 / 0$ interface at the applied strain $\epsilon_{\mathrm{o}}=0.0007689$. The results for normal stresses $\sigma_{x x}, \sigma_{y y}$, and $\sigma_{z z}$ along the midplane of the laminate at $Z=h$ for $\epsilon_{o}=0.001$, presented in Figures $12-14$, respectively, confirm the fact that there is no damage development in the $0^{\circ}$ layer. Clearly, the stresses $\sigma_{z z}$ 


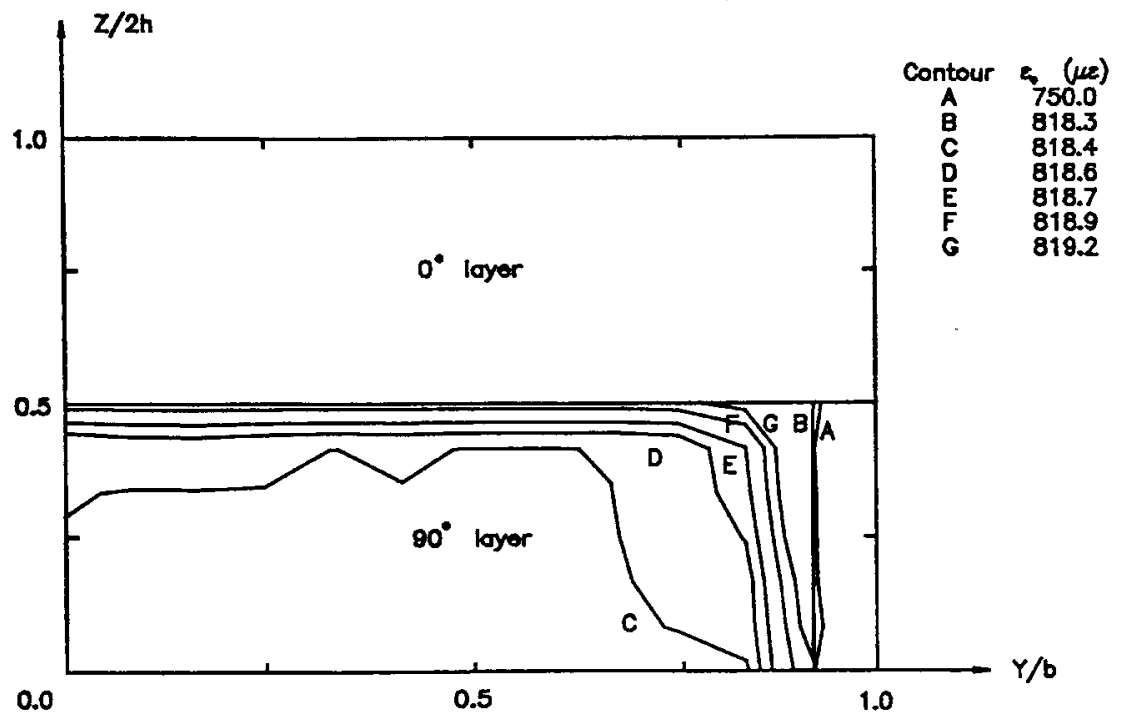

Figure 4. Growth of damage zone in $\left[0 / 90 \%\right.$ s laminate with various axial strains $\epsilon_{0}$.

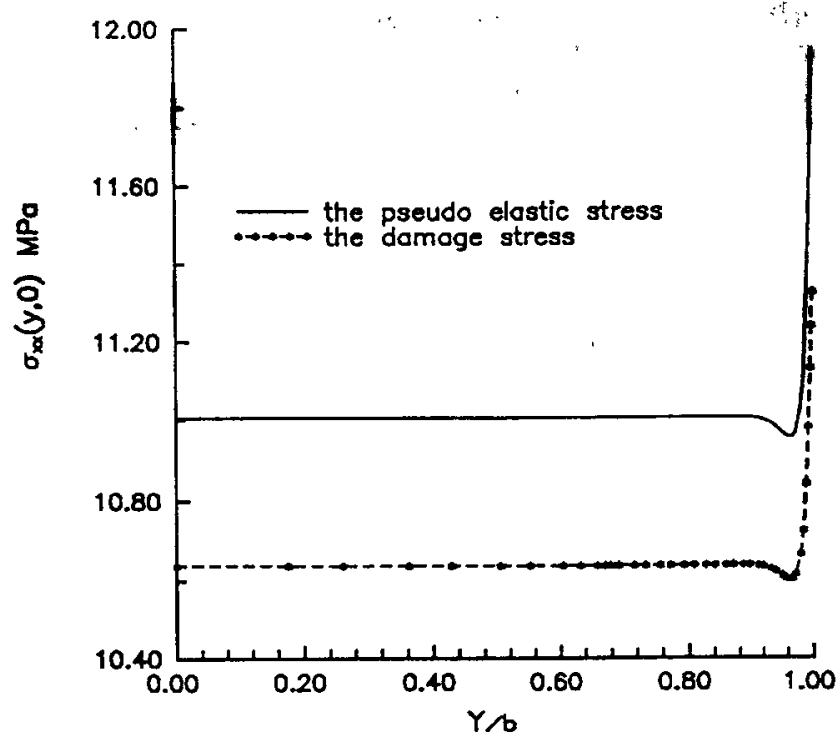

Figure 5. The $\sigma_{x x}$ along the midplane of $\left[0 / 90^{\circ}\right]_{\mathrm{s}}$ laminate. 


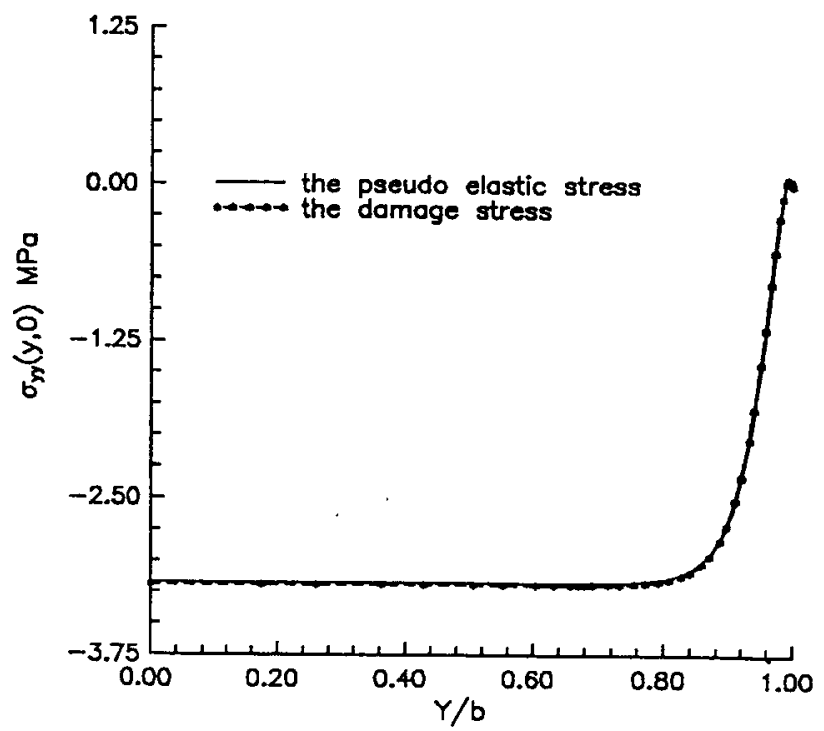

Figure 6. The $\sigma_{\mathrm{yy}}$ along the midplane of $\left[0 / 90^{\circ}\right]_{\mathrm{s}}$ laminate.

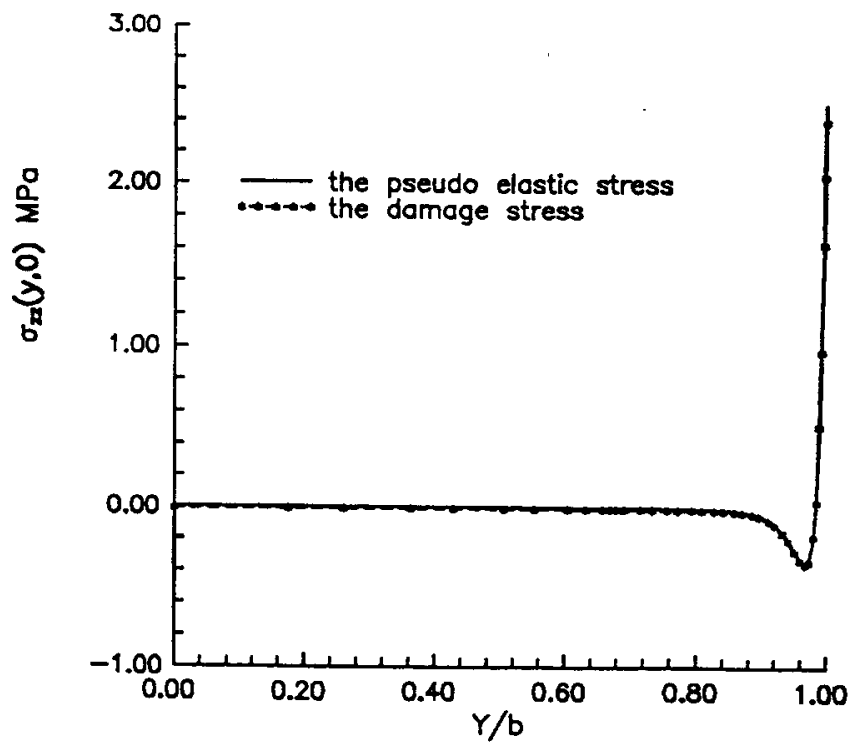

Figure 7. The $\sigma_{\mathrm{zz}}$ along the midplane of $[0 / 90 \%]_{\mathrm{s}}$ laminate. 


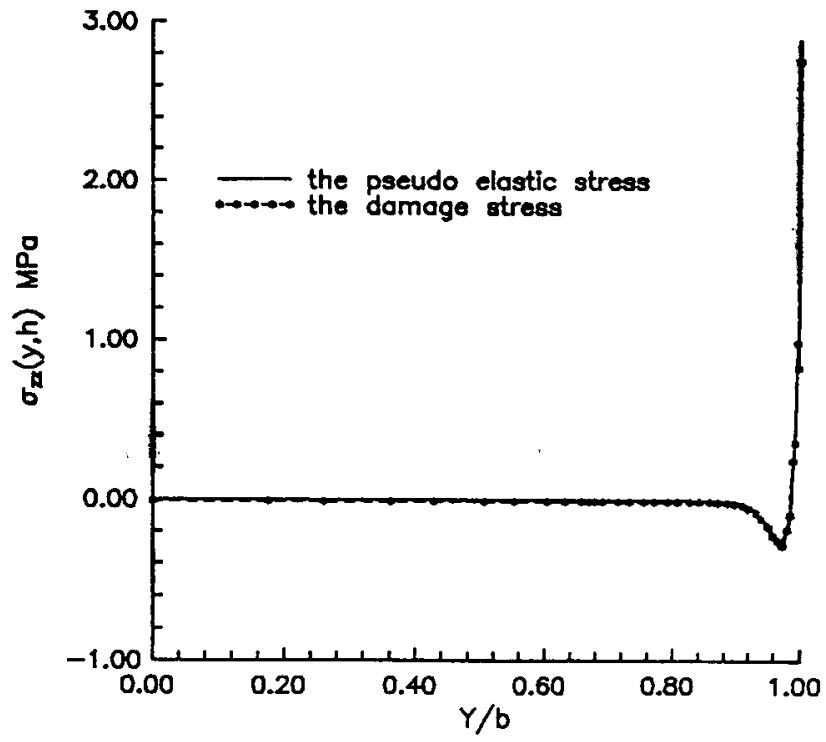

Figure 8. The $\sigma_{z z}$ along the interface of $\left[0 / 90^{\circ}\right]_{\mathrm{s}}$ laminate.

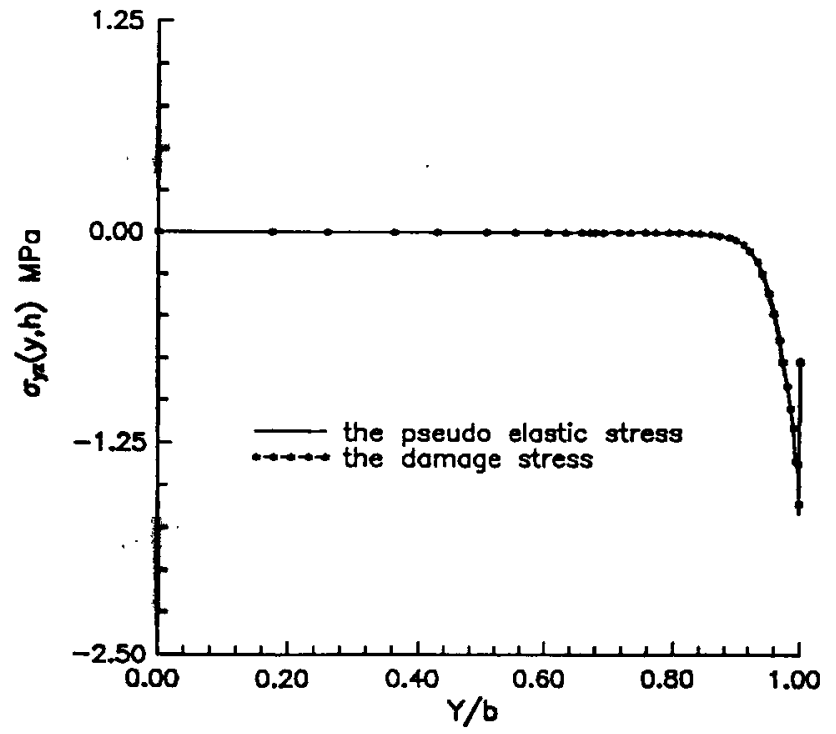

Figure 9. The $\sigma_{\mathrm{yz}}$ along the interface of $\left[0 / 90^{\circ}\right]_{\mathrm{s}}$ laminate. 


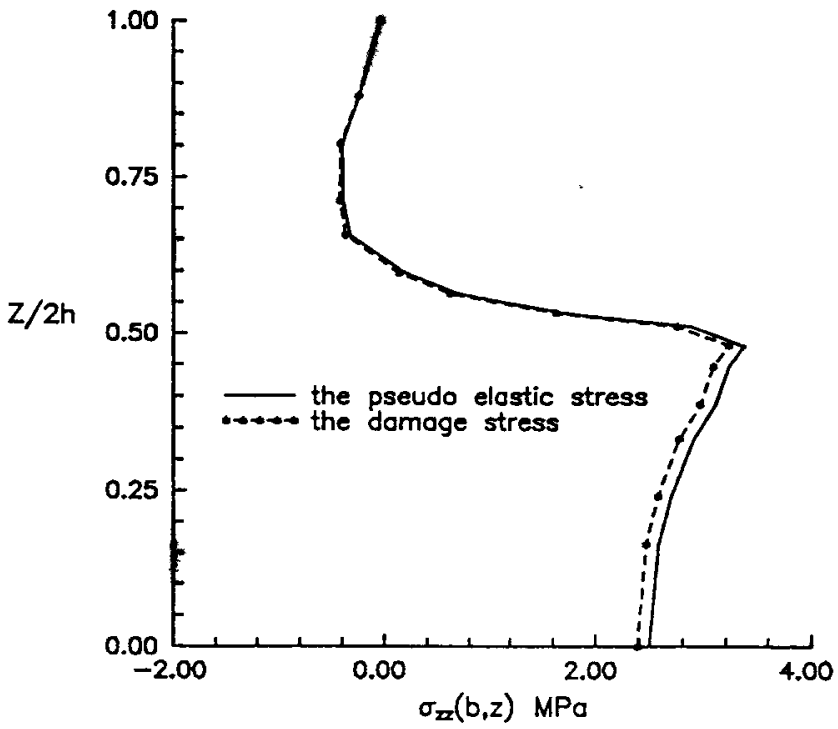

Figure 10. The $\sigma_{\mathrm{zz}}$ along the free edge of $\left[0 / 90^{\circ}\right]_{\mathrm{s}}$ laminate.

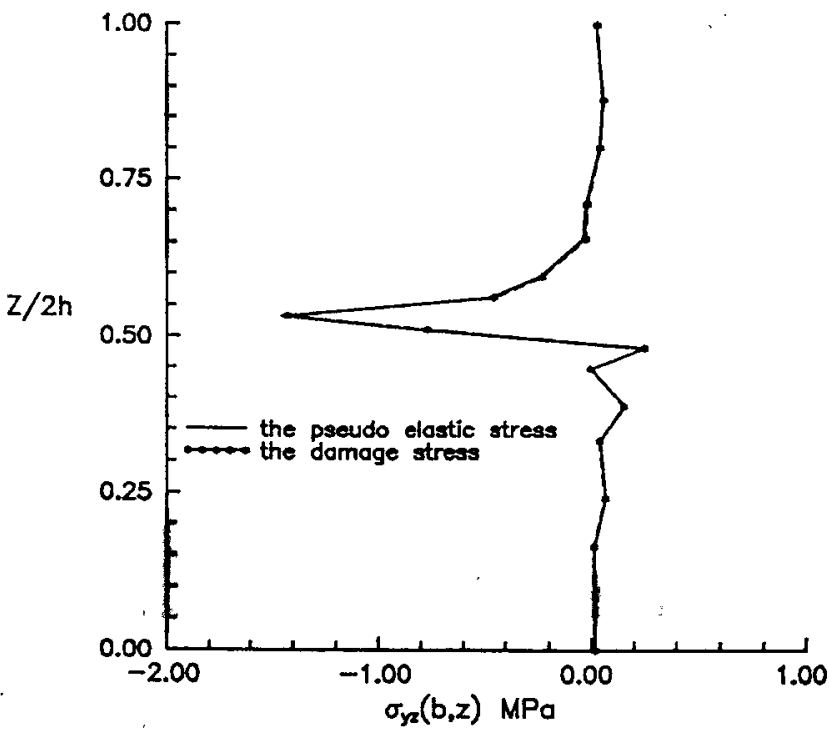

Figure 11. The $\sigma_{\mathrm{yz}}$ along $\mathrm{y} / \mathrm{b}=0.9993$ in $\left[0 / 90^{\circ}\right]_{\mathrm{s}}$ laminate. 


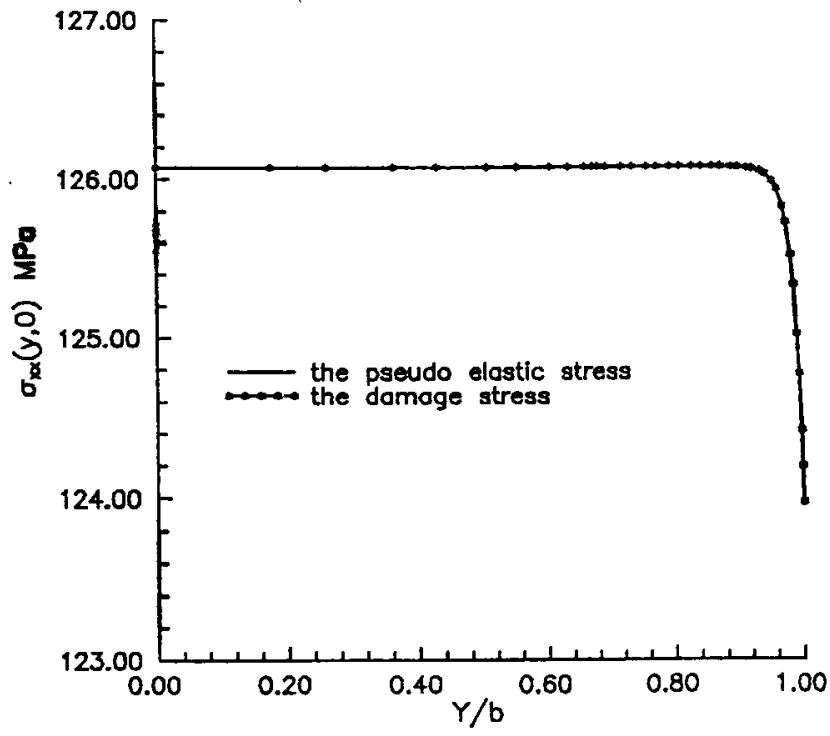

Figure 12. The $\sigma_{\mathrm{xx}}$ along the midplane of $[90 / 0 \%]_{\mathrm{s}}$ laminate.

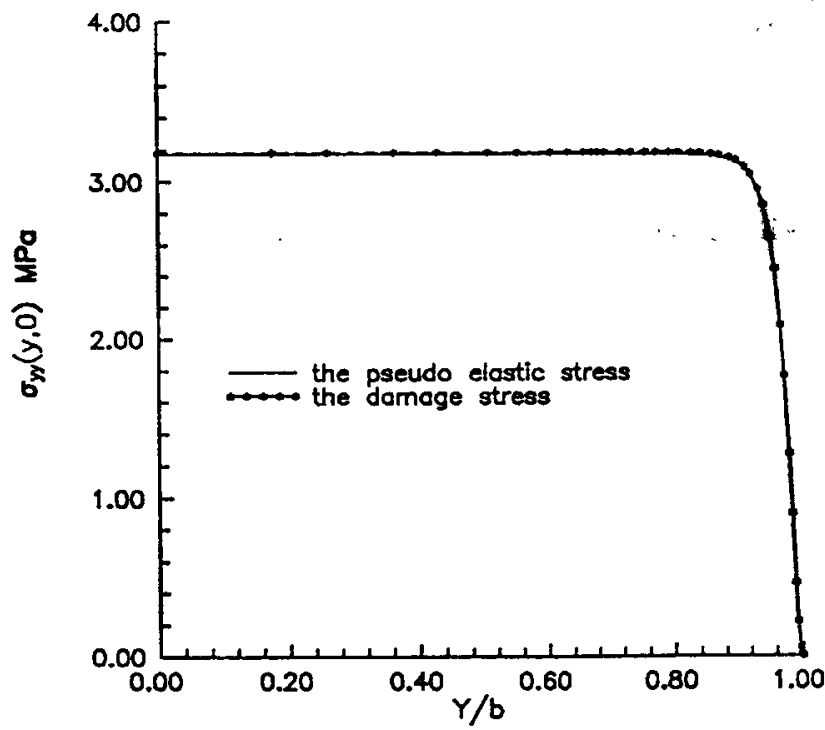

Figure 13. The $\sigma_{y y}$ along the midplane of $[90 / 0 \%]_{\mathrm{s}}$ laminate. 


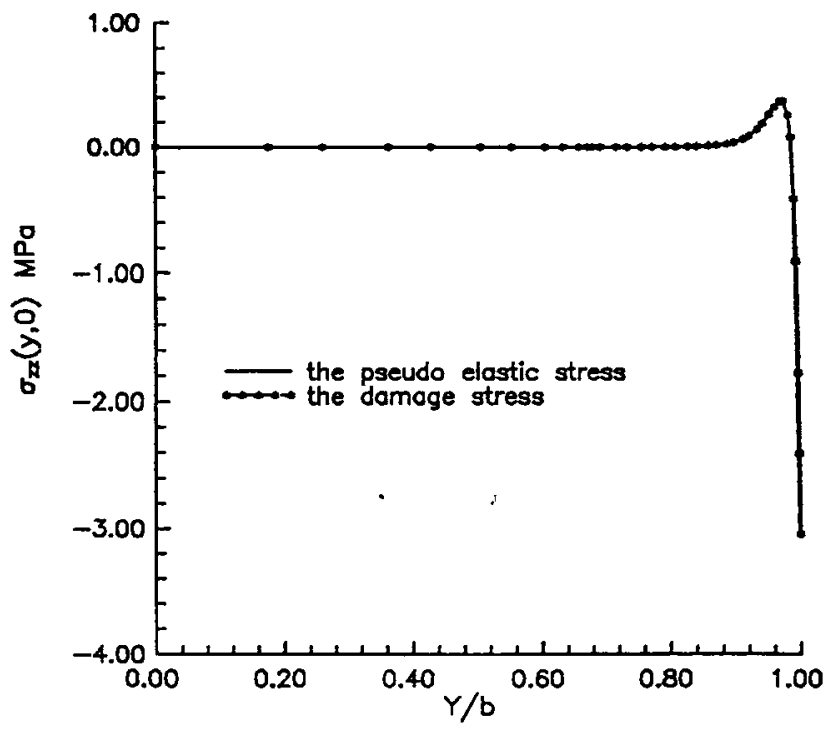

Figure 14. The $\sigma_{\mathrm{zz}}$ along the midplane of $\left[90 / 0^{\circ}\right]_{\mathrm{s}}$ laminate.

along the midplanes of the two laminates, $\left[90 / 0^{\circ}\right]_{s}$ and $\left[0 / 90^{\circ}\right]_{s}$, are of opposite sign. However, the stress distributions are not exactly mirror images because of different three-dimensional effects near the free edges of the two laminates. Displayed in Figure 15 and Figure 16 are the distributions of stresses $\sigma_{z z}$ and $\sigma_{y z}$ along the interface and in Figure 17 and Figure 18, along the free edge of the laminate for $\epsilon_{o}=0.001$, respectively. These stress components in the $90^{\circ}$ layer only are affected, which are similar to those in the previous case, revealing that the presence of the $90^{\circ}$ graphite/epoxy composite lamina has properties that can cause decreasing stiffness with increasing tensile strain due to material damage. The growth of damage zones in $\left[90 / 0^{\circ}\right]_{s}$ laminate is illustrated in Figure 19.

The extensions of damage zones for the $\left[90 / 0^{\circ}\right]_{s}$ laminate shown in Figure 19 are quite different from those for the $\left[0 / 90^{\circ}\right]_{s}$ case shown in Figure 4 . This difference can be explained by observing the distributions of stress $\sigma_{z z}$ in the two laminates; for these cross-ply laminates, the in-plane shear stress component is absent, the sign of out-of-plane shear stress does not influence the damaging process, and the values of stress $\sigma_{22}$, i.e., $\sigma_{x x}$ in the $90^{\circ}$ layer, are positive for the two laminates when they are subjected to uniaxial tension. As shown in Figure 8, the compressive stress $\sigma_{x z}$ in the $\left[0 / 90^{\circ}\right]_{s}$ laminate is induced in the region from $Y / b=0.827$ to 0.985 . The negative value of stress $\sigma_{z z}$ (or $\sigma_{33}$ ) contributes a negative effect on the damage function from the coupling term $R_{23} \cdot \sigma_{22} \cdot \sigma_{33}$. Therefore, no damage is expected to initiate within this area below a comparatively low applied strain level, although this region will experience damage process at a higher applied strain. 


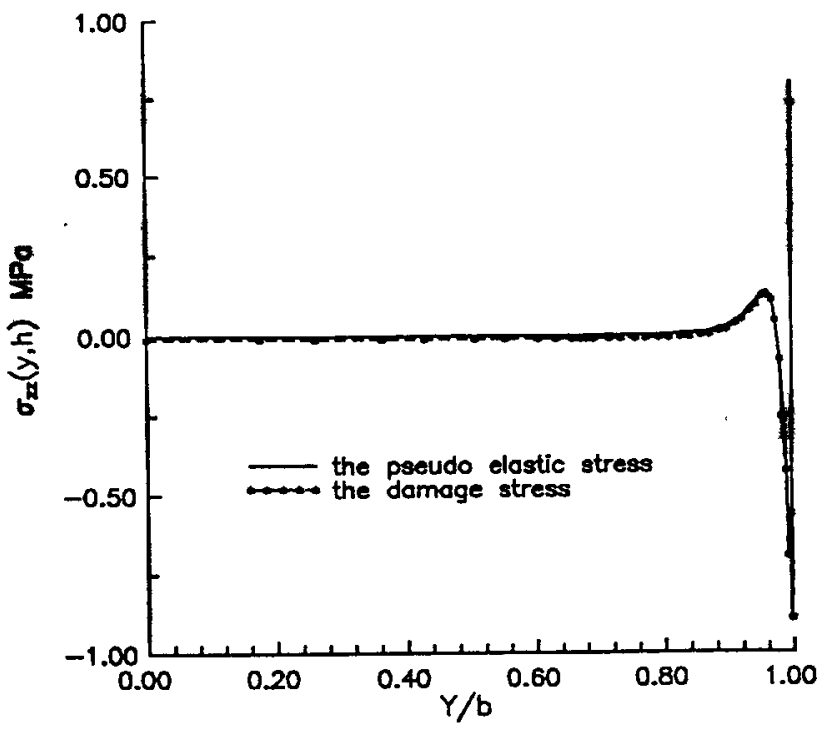

Figure 15. The $\sigma_{22}$ along the interface of $\left[90 / 0^{\circ}\right]_{\mathrm{s}}$ laminate.

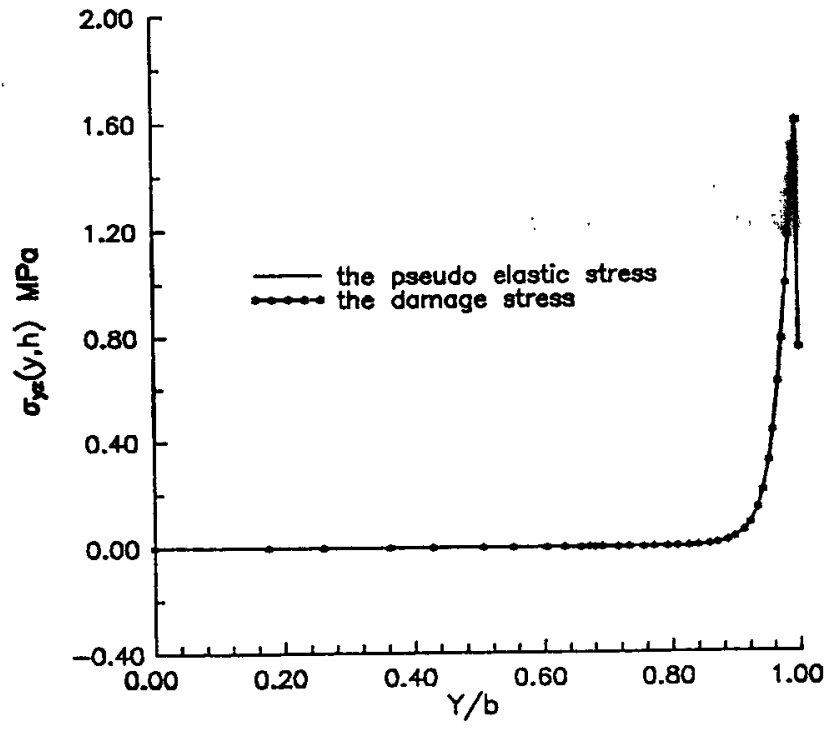

Figure 16. The $\sigma_{\mathrm{yz}}$ along the interface of $\left[90 / 0{ }^{\circ}\right]_{\mathrm{S}}$ laminate. 


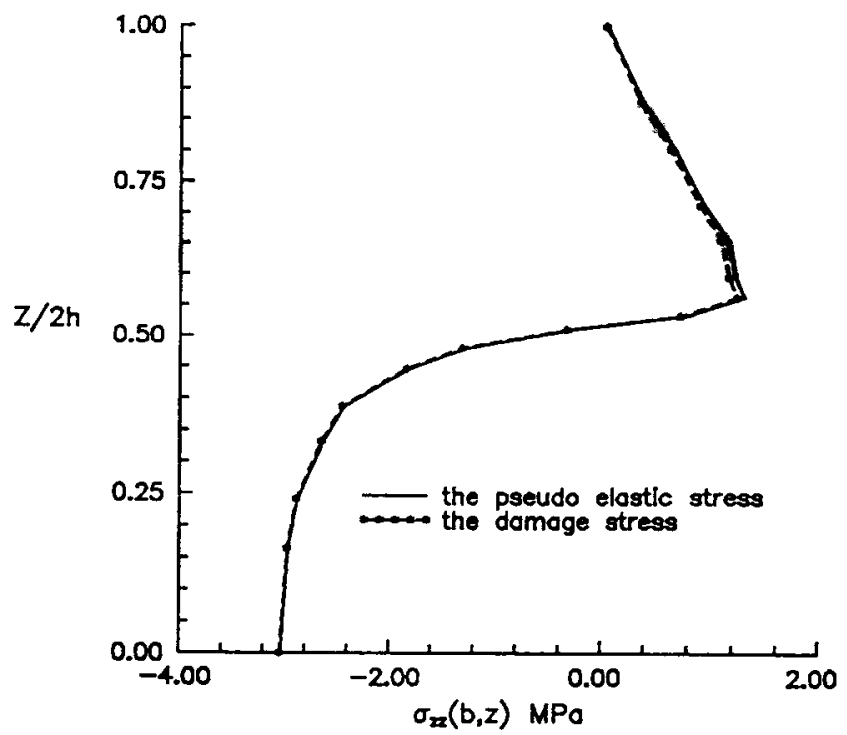

Figure 17. The $\sigma_{\mathrm{zz}}$ along the free edge of $\left[90 / 0^{\circ}\right]_{\mathrm{s}}$ laminate.

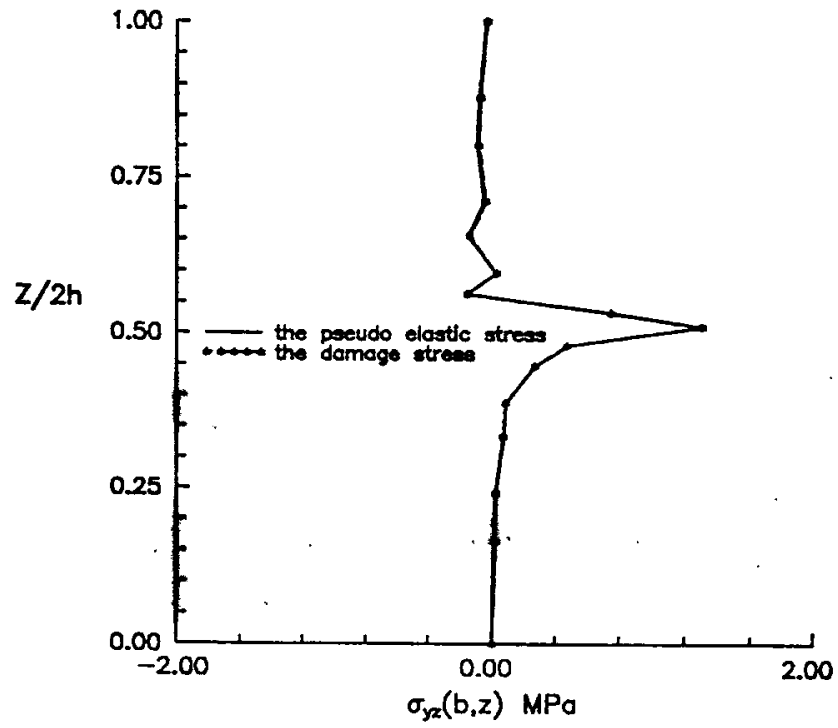

Figure 18. The $\sigma_{\mathrm{yz}}$ along $\mathrm{y} / \mathrm{b}=0.9993$ in $\left[90 / 0^{\circ}\right]_{\mathrm{s}}$ laminate. 


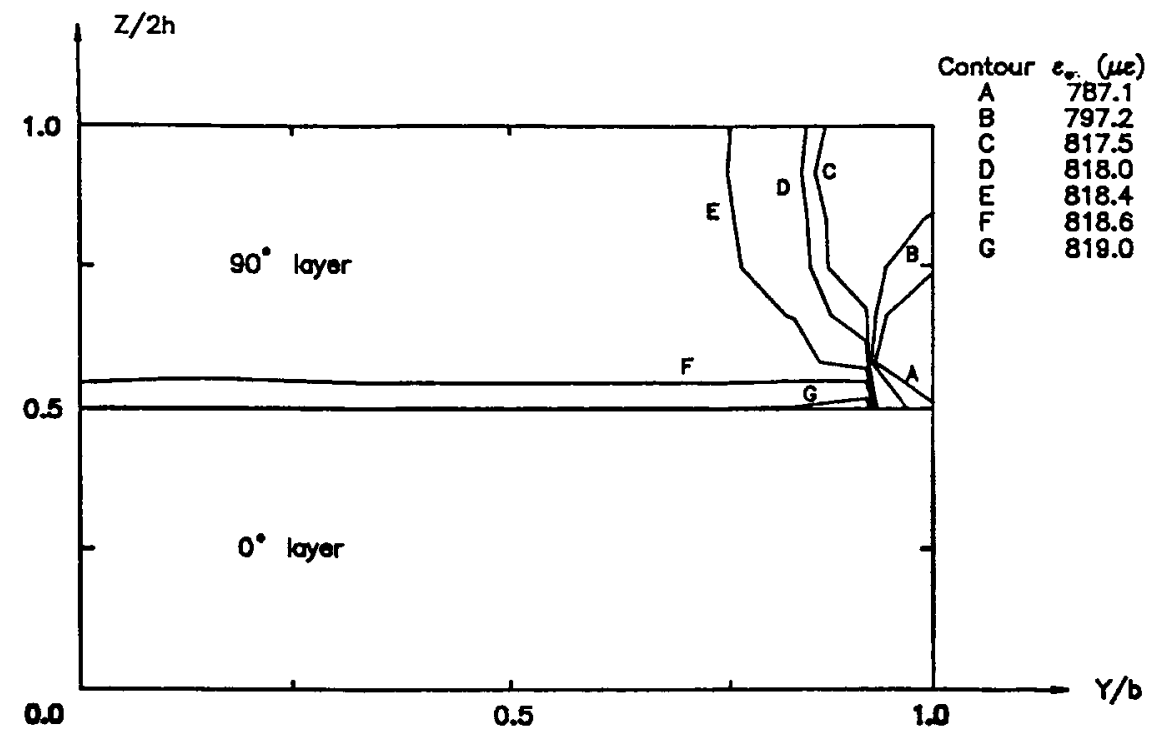

Figure 19. Growth of damage zone in $\left[90 / 0^{\circ}\right]_{\mathrm{s}}$ laminate with various axial strains $\epsilon_{\circ}$.

The angle-ply laminates under consideration are composed of an equal number of layers, with fibers oriented at $+\theta$ and $-\theta$ to the loading direction. Analyses of the behaviors of these balanced and symmetric laminates provide fundamental insight into the influence of fiber orientation on interlaminar stresses. As an example, consider first the $\left[ \pm 45^{\circ}\right]_{s}$ laminate, which induces mismatch in elastic shear properties.

The development of the damage zone in the $\left[ \pm 45^{\circ}\right]_{s}$ laminate is rather different from those in the cross-ply laminates described above. The damage initiates in the elements along the interface near the free edge but not at the free edge at $\epsilon_{o}=0.000531$, and the damage zone then extends symmetrically into the $45^{\circ}$ and $-45^{\circ}$ plies. Figure 20 displays the growth of damage zones in the laminate. The results of the damaged and pseudo elastic stress distributions under the tensile strain $\epsilon_{o}=0.001$ for the $\left[ \pm 45^{\circ}\right]_{s}$ laminate are included in Figures 21-26. The distributions of stress components $\sigma_{x x}, \sigma_{y y}, \sigma_{z z}$, and $\sigma_{x z}$ along the $45 /-45$ interface are plotted in Figures 21-24, respectively. It can be seen from the figures that the maximum magnitudes of stresses $\sigma_{x x}, \sigma_{y y}$, and $\sigma_{x z}$ are reduced due to damage. However, the peak value of damaged stress $\sigma_{z z}$ in Figure 23 is higher than that of the pseudo elastic one. This is caused by the material anisotropy in $\pm 45^{\circ}$ laminae under off-axis tension, unlike those in the cross-ply laminates, which are orthotropic in nature. The free edge stress distributions, $\sigma_{z z}$ and $\sigma_{x z}$, for the damaged and pseudo elastic cases, are illustrated in Figure 25 and Figure 26, respectively. Symmetric stress distribution of $\sigma_{x z}$ about the interface can be observed due to the specific configuration of the laminate. 


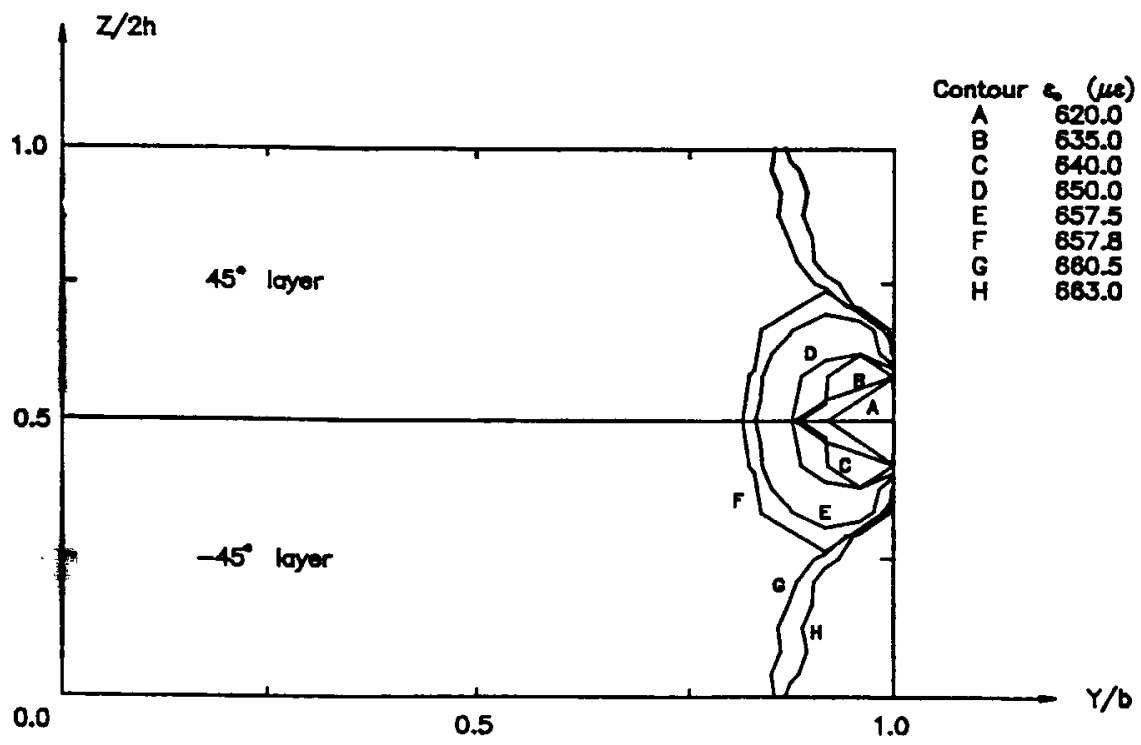

Figure 20. Growth of damage zone in $\left[45 /-45 \%_{\mathrm{s}}\right.$ laminate with various axial strains $\epsilon_{\mathrm{o}}$.

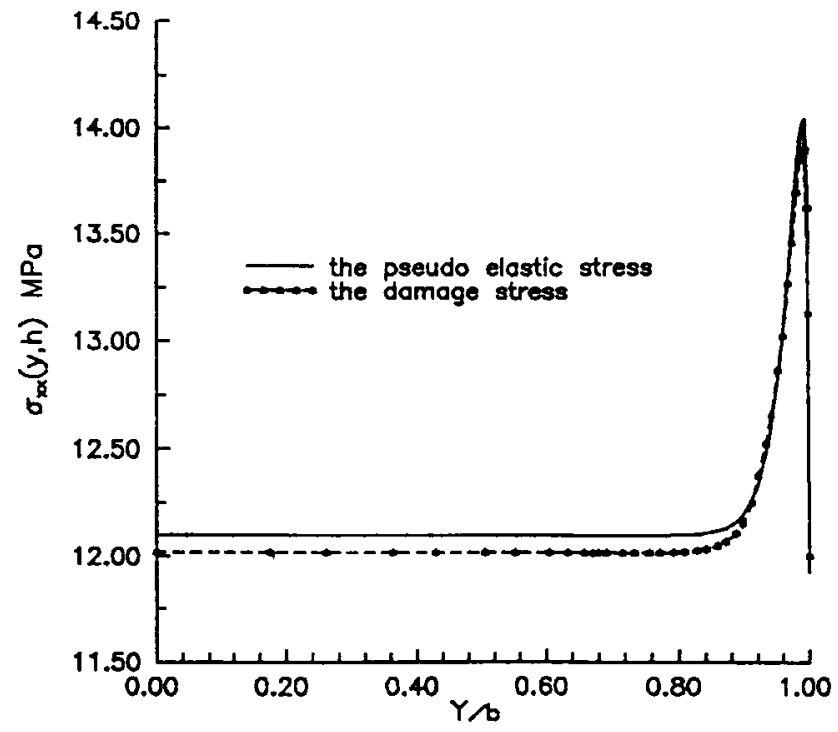

Figure 21. The $\sigma_{\mathrm{xx}}$ along the interface of $\left[45 /-45^{\circ}\right]_{\mathrm{s}}$ laminate. 


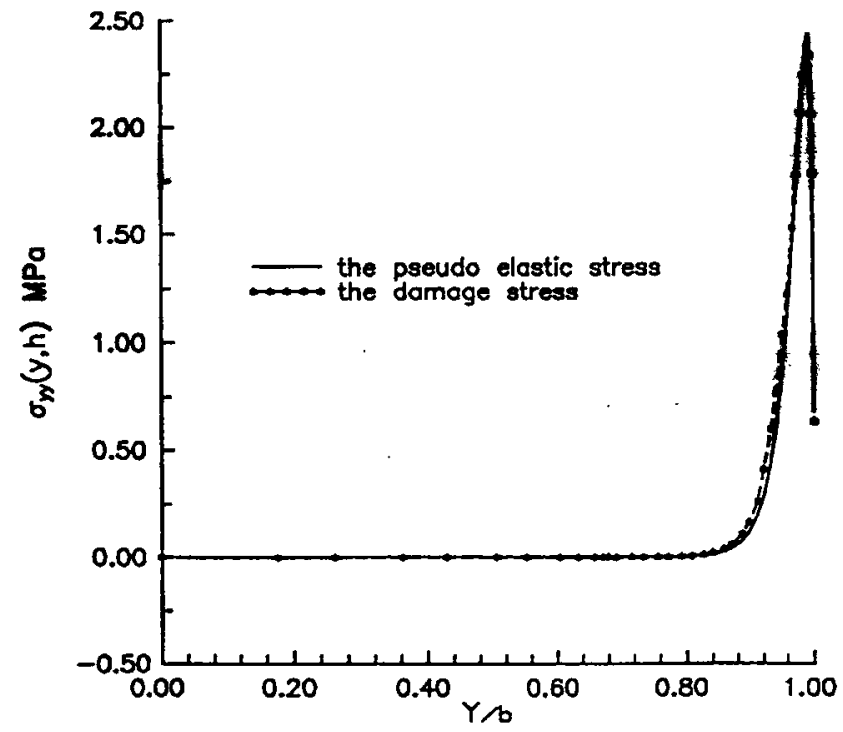

Figure 22. The $\sigma_{\mathrm{yy}}$ along the interface of $\left[45 /-45^{\circ}\right]_{\mathrm{s}}$ laminate.

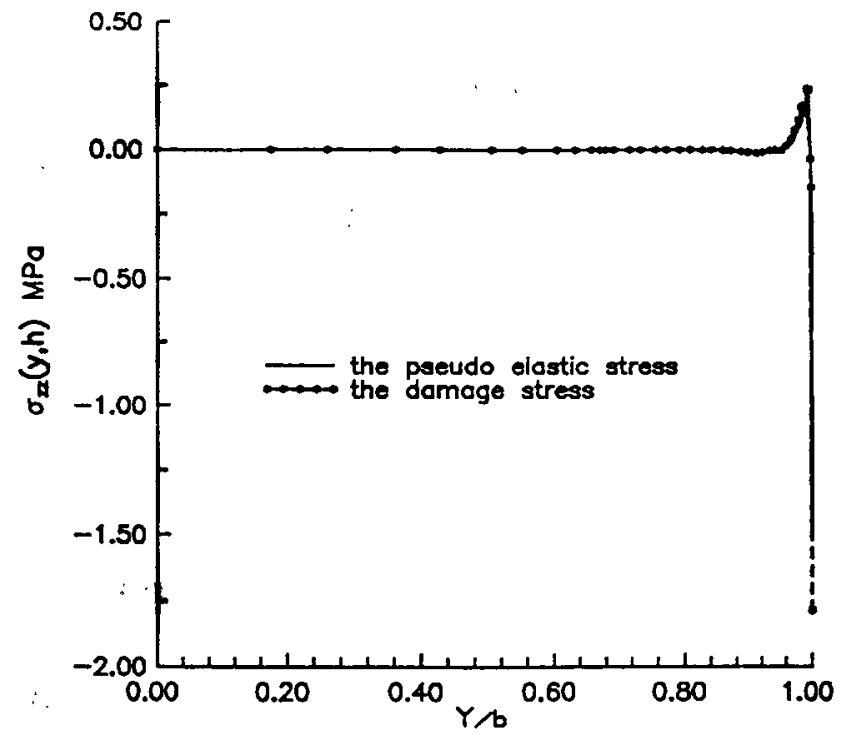

Figure 23. The $\sigma_{z z}$ along the interface of $\left[45 /-45^{\circ}\right]_{s}$ laminate. 


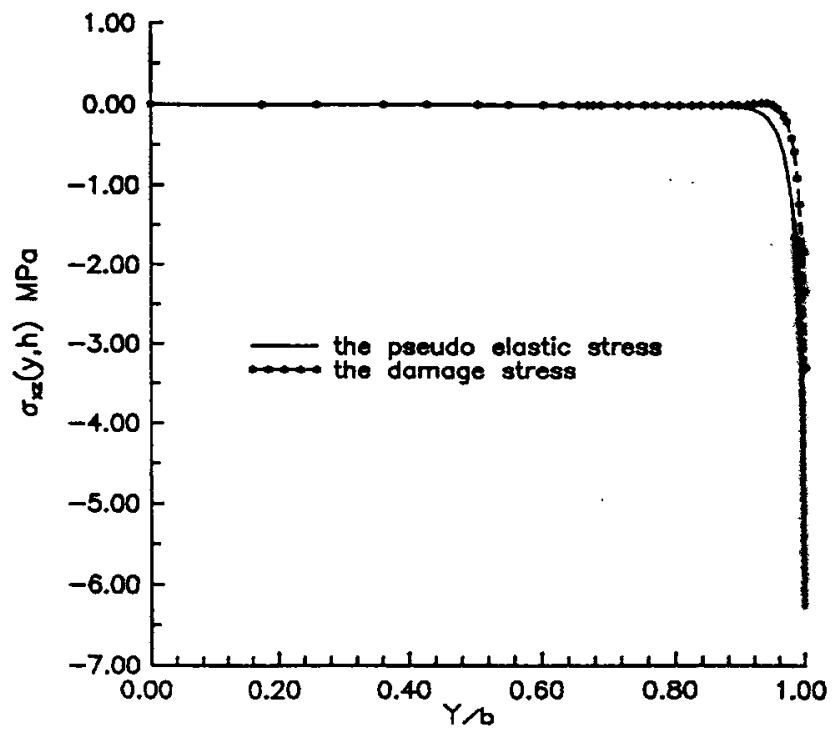

Figure 24. The $\sigma_{\mathrm{xz}}$ along the interface of $\left[45 /-45^{\circ}\right]_{\mathrm{s}}$ laminate.

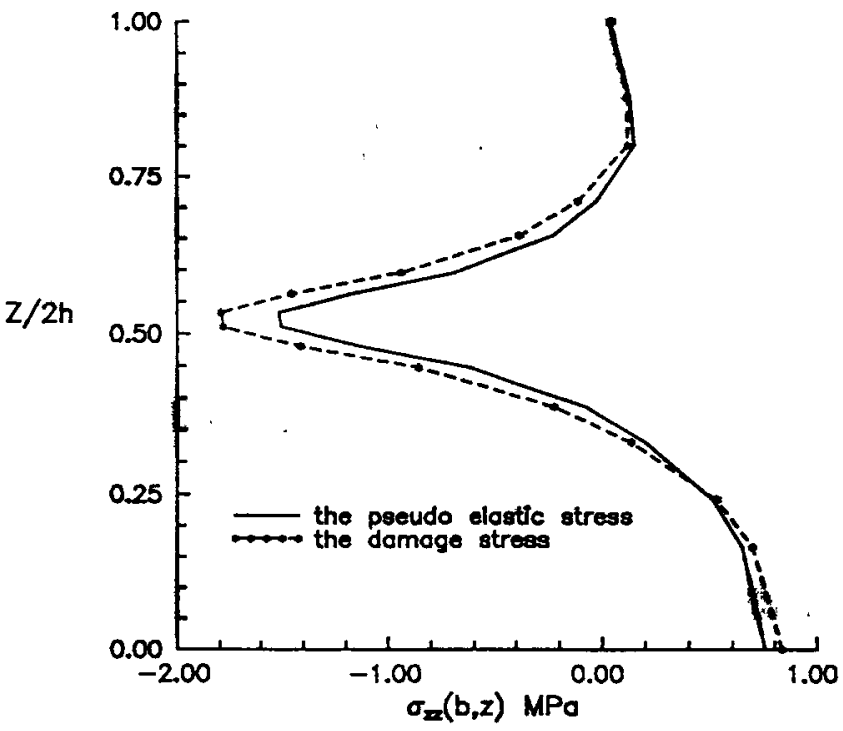

Figure 25. The $\sigma_{z z}$ along the free edge of $\left[45 /-45^{\circ}\right]_{5}$ laminate. 


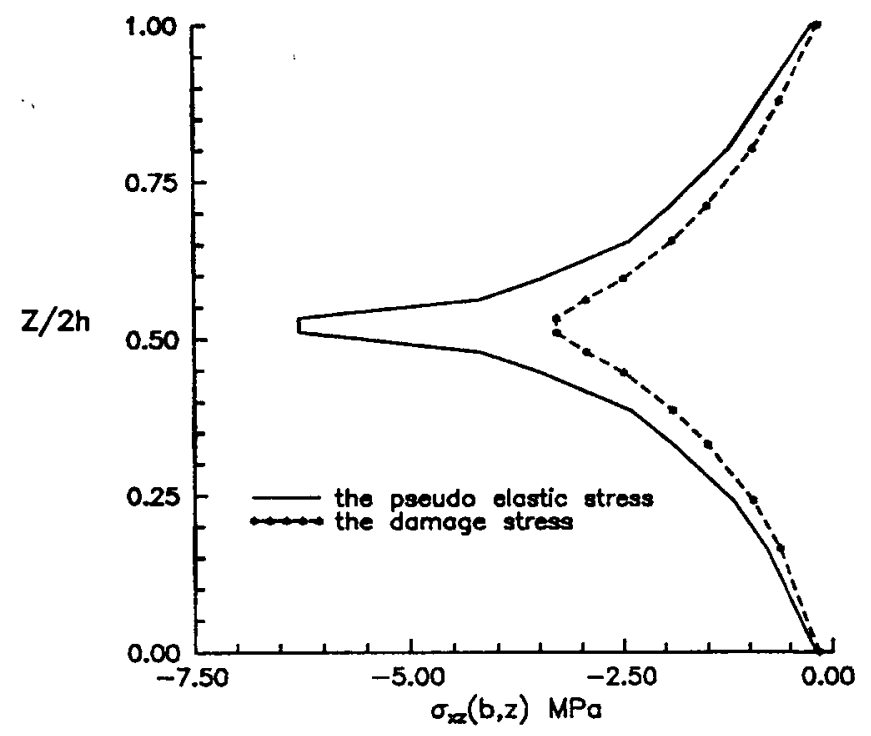

Figure 26. The $\sigma_{\mathrm{xz}}$ along the free edge of $\left[45 /-45^{\circ}\right]_{\mathrm{s}}$ laminate.

\section{CONCLUSIONS}

The constitutive model for the damage behavior of laminated composite materials has been established in such a way that it can be easily incorporated into finite element analysis, which is important in characterizing the damage response of composite structures. The formulation of the elastic-damaged analysis for composite laminates with straight edges subjected to the loading of in-plane strain using a quasi-three-dimensional finite element approach has been performed. The distributions of the interlaminar damage stress and pseudo elastic stress are given for the $\left[0 / 90^{\circ}\right]_{s},\left[90 / 0^{\circ}\right]_{s}$, and $\left[ \pm 45^{\circ}\right]_{s}$ graphite/epoxy composite laminates. The developments of damage zones in the laminates are also demonstrated.

It has been shown that the nonlinear material properties of the graphite/epoxy composites induced by the damage effects are important factors to be included in achieving an accurate stress analysis. The availability of accurate stress distributions throughout the composite laminates makes it possible to predict the location and mode of material damage, which are dependent upon the fiber orientations and stacking sequences of the laminates, with the aid of proper failure criteria.

\section{REFERENCES}

1. Reifsnider, K. L., ed. 1982. "Damage in Composite Materials: Basic Mechanisms, Accumulation, Tolerance, and Characterization," ASTM STP-775.

2. Schapery, R. A. 1988. "A Method for Mechanical State Characterization of Inelastic Composite Laminates with Damage," Report No. MM5762-88-10, Texas A\&M Univ. 
3. Schapery, R. A. 1990. "A Theory of Mechanical Behavior of Elastic Media with Growing Damage and Other Changes in Structure," J. Mech. Phys. Solids, 38:215-253.

4. Chow, C. L. and F. Yang. 1992. "A Simple Model for Brittle Composite Lamina with Damage," $J$. of Reinforced Plastics and Composites, 11(3):222-242.

5. Chow, C. L., F. Yang and A. Asundi. 1992. "A Method of Continuum Damage Analysis for Anisotropic Materials and Its Application to Composite Laminates," Int. J. of Damage Mechanics, l(3):347-366.

6. Pipes, R. B. and N. J. Pagano. 1970. "Interlaminar Stress in Composite Laminates under Uniform Axial Extension," J. Composite Materials, 4:538-548.

7. Tang, S. and A. Leavy. 1975. "A Boundary Layer Theory-Part II: Extension of Laminated Finite Strip," J. Composite Materials, 9:42-52.

8. Hsu, P. W. and C. T. Herakovich. 1977. "Edge Effects in Angle-Ply Composite Laminates," J. Composite Materials, 11:422-432.

9. Wang, S. S. and I. Choi. 1982. "Boundary-Layer Effects in Composite Laminates: Part 1 \& 2," Trans. ASME, J. Appl. Mech., 49:541-548.

10. Pagano, N. J. 1978. "Stress Fields in Composite Laminates," Int. J. Solids Structures, 14:385-400.

11. Pagano, N. J. and S. R. Soni. "Global-Local Laminate Variational Model," Int. J. Solids Structures, 19:207-228.

12. Pinchas, Bar-Yoseph and Givon Siton. 1985. "The Effect of Material Non-Linearity on the Interlaminar Stress Field in Composite Laminates," Computers \& Structures, 21:1105-1118.

13. Wang, A. S. D. and F. W. Crossman. 1977. "Some New Results on Edge Effects in Symmetric Composite Laminates," J. Composite Materials, 11:92-106.

14. Whitcomb, J. D., I. S. Raju and J. G. Goree. 1982. "Reliability of the Finite Element Method for Calculating Free Edge Stresses in Composite Laminates," Computers \& Structures, 15:23-37.

15. Icardi, Ugo and Andrea Manuello. 1988. "Free Edge Stress Propagation in Composite Laminates," Computers \& Structures, 29:365-380.

16. Spilker, R. L. and S. C. Chou. 1980. "Edge Effects in Symmetric Composite Laminates: Importance of Satisfying the Traction-Free Edge Condition," J. Composite Materials, 14:2-20.

17. Spilker, R. L. 1980. "A Traction-Free-Edge Hybrid-Stress Element for the Analysis of Edge Effects in Cross-Ply Laminates," Computers \& Structures, 12:167-179.

18. Talreja, R. 1989. "Damage Development in Composites: Mechanisms and Modeling," J. Strain Analysis, 24:215-222.

19. Allen, D. H., C. E. Harris and S. E. Groves. 1987. "A Themomechanical Constitutive Theory for Elastic Composites with Distributed Damage: Part I \& II," Int. J. Solids Structures, 23:1301-1318, 1319-1338.

20. Ledeveze, P. et al. 1982. "Damage and Fracture of Tridirectional Composite," Proc. ICCM-IV, pp. 619-628.

21. Allix, O. et al. 1987. "Damage Mechanics for Composite Laminates under Complex Loading," Proc. IUTAM/IUM, Symposium Yielding, Damage and Failure of Anisotropic Solids, Grenoble.

22. Bathe, K. J. 1982. Finite Element Procedures in Engineering Analysis. Englewood Cliffs, NJ: Prentice-Hall. 